\title{
Screening and Identification of Differentially Expressed and Adipose Growth-Related Protein-Coding Genes During the Deposition of Perirenal Adipose Tissue in Rabbits
}

This article was published in the following Dove Press journal:

Diabetes, Metabolic Syndrome and Obesity: Targets and Therapy

\author{
Guoze Wang ${ }^{1,2, *}$ \\ Kun $\mathrm{Du}^{\mathrm{l}}$,* \\ Zhenjian Xie $\mathbb{D D}^{3}$ \\ Renyong Tang ${ }^{3}$ \\ Xianbo Jia' \\ Shiyi Chen' \\ Songjia Lai'
}

'Farm Animal Genetic Resources Exploration and Innovation Key Laboratory of Sichuan Province, Sichuan Agricultural University, Chengdu 611130 , People's Republic of China; ${ }^{2}$ College of Food Science, Guizhou Medical University, Guiyang 550025, People's Republic of China; ${ }^{3}$ College of Pharmacy and Biological Engineering, Chengdu University, Chengdu 610106, People's Republic of China

*These authors contributed equally to this work
Correspondence: Songjia Lai Email laisj5794@I63.com

\begin{abstract}
Background: Rabbit is a good model for genetic and medical studies in other livestock species. The rabbit shows low adipose tissue deposition, and the phenomena indicates that there is some specificity of adipose deposition during the rabbit growth. However, little is known about genes that regulate the growth of adipose tissue in rabbits.

Materials and Methods: Deep RNA-seq and comprehensive bioinformatics analyses were used to characterize the genes of rabbit visceral adipose tissue (VAT) at 35, 85 and 120 days after birth. Differentially expressed genes (DEGs) were identified at the three growth stages by DESeq. To explore the function of the candidate genes, Gene Ontology (GO) enrichment and Kyoto Encyclopedia of Genes and Genomes (KEGG) pathway analyses were performed. Six DEGs were randomly selected, and their expression profiles were validated by q-PCR.

Results: A total of 20,303 known transcripts and 99,199 new transcripts from 8 RNA sequencing libraries were identified, and 34 differentially expressed genes (DEGs) were screened. GO enrichment and KEGG pathway analyses revealed that the DEGs were mainly involved in lipid metabolism regulation including acylglycerol metabolic process and mobilization, and decomposition of lipids to generate ATP in adipocytes and fatty acid metabolism, included LOC100342322 and LOC100342572. In addition, 133 protein-coding genes that play a role in adipose growth and development were screened, including acyl-CoA synthetase longchain family member 5 (ACSL5) and fatty acid-binding protein 2 (FABP2). The validation results of six DEGs by q-PCR showed similar trends with the results of RNA-seq.

Conclusion: In summary, this study provides the first report of the coding genes profiles of rabbit adipose tissue during different growth stages. These data allow for the identification of candidate genes for subsequent studies on rabbit genetics and regulation of adipose cells, and provide an animal model for studying obesity in humans.
\end{abstract}

Keywords: rabbit, RNA-seq, adipose tissue, growth, protein-coding gene, fat deposition

\section{Background}

As an important economic and agricultural animal, rabbits are bred for meat, fur production, companionship as pets, and also for use in biomedical research. Total world production of rabbit meat is about 1.4 million tonnes across Asia, Europe, Africa and Americas. ${ }^{1}$ Rabbit is a good model for genetic and medical studies in other livestock species due to its short generation interval and the low cost of its carcass. ${ }^{2}$ For example, injection of injured rabbit vocal folds with human adipose-derived stem 
cells (ADSCs) improves wound healing and shows fewer signs of scarring, ${ }^{3}$ and the injection of ADSCs into injured lumbar discs in rabbit models could be an effective treatment for degenerative disc disease. ${ }^{4}$ In the rabbit model, ADSCs alleviate osteoporosis by enhancing osteogenesis, inhibiting adipogenesis, ${ }^{5}$ and adhering to defective cartilage that cannot promote cartilage regeneration. ${ }^{6}$

Visceral adipose tissue is a very important adipose tissue in the body, ${ }^{7}$ mainly composed of white adipose cells, as an important energy storage organ and secretory organ in the body. ${ }^{8,9}$ At present, some studies ${ }^{10,11}$ have shown that the increase in the size and number of adipocytes in visceral adipose tissue are closely related to metabolic syndrome such as obesity. Therefore, studies on visceral adipocyte differentiation and its potential regulatory mechanisms have long been the core of obesity research. When the rabbit is born, the visceral fat cells are mainly at the precursor adipocyte stage. As the rabbit ages, cell morphology changes from fibrous to round, and the intracellular lipid droplets gradually appear and form a large number of small lipid droplets. After terminal differentiation, the cells are filled with a large lipid droplet to form mature single-compartment adipocytes. ${ }^{12,13}$ Since the adipose tissue in rabbits has a lower deposition rate during growth, rabbits can serve as an ideal model system to study adipose regulation. , $^{5,14}$

RNA-seq has emerged as a powerful tool for analyzing gene expression patterns and changes. ${ }^{16}$ Owing to the advantages of low background signals, high accuracy, broader genome coverage, high sensitivity and lower amounts of samples required, RNA-seq is increasingly used for profiling global gene expression patterns. ${ }^{16}$ Wang et $\mathrm{al}^{17}$ analyzed RNA sequences from the longissimus dorsi muscle of two indigenous Chinese pig breeds and two introduced pig breeds, identifying 85 genes related to muscle growth and 27 genes related to lipid deposition. Similar studies have been reported in cattle, ${ }^{18,19}$ sheep, ${ }^{20,21}$ chickens $^{22}$ and other domestic animals. However, little information exists on genes that regulate the growth of adipose tissue in rabbits.

Hence, we performed deep RNA-sequencing during three important stages of adipose deposition $(35,85$, and 120-days post-birth) to screen and identify the genes that involved in regulating adipose growth in rabbits. The results of this study may provide references for future rabbit genetic and breeding research, and further establish rabbits as a model organism for studying human obesityrelated diseases.

\section{Materials and Methods}

\section{Animal Preparation and Adipose Tissue Collection}

Given the plasticity and maturation of rabbit visceral adipose tissue, ${ }^{23} 35,85$, and 120 days old Tianfu Black rabbits (native species in Sichuan province of China) were used and weighed in our study. They were obtained in teaching experiment rabbit farm in Ya'an campus of Sichuan Agricultural University in Sichuan, China. All rabbits used (all were male) were raised under the condition with the same diet, environmental temperature and given free access to water and food. In this study, rabbits were euthanized using electrical stunning and exsanguination. Three biological replicates of perirenal adipose were collected for 35-day (YR) and 120-day (TR), and two were collected for 85-day (MR). The samples were stored at $-80^{\circ} \mathrm{C}$ until RNA extraction.

\section{Preparation of Paraffin Section and Hematoxylin and Eosin Staining}

After the adipose samples were fixed with $4 \%$ paraformaldehyde solution for 12 to 24 hours, excess water was removed using a full-automatic dehydrator (Leica, ASP6025, Germany). After being soaked in paraffin for $30 \mathrm{~min}$, the mixture was transferred into a mold filled with liquid paraffin, cooled, and shaped. The block was sliced by microtome (Leica, KH-Q300, Germany), and stained with hematoxylin and eosin (HE). The dyed slices were dehydrated with different gradients of alcohol and sealed by dropping the sealing gum. Finally, the slice images were collected using a biological microscope (Olympus, CX31-32C02, Japan).

\section{The RNA Extraction, Quality Testing, Library Construction and Sequencing}

Total RNA was extracted from 50 to $60 \mathrm{mg}$ of perirenal adipose tissue in each sample by using the Trizol Reagent (Life Technologies, Carlsbad, CA, USA) according to the manufacturer's instructions and dissolved in RNase-free water. The purity and integrity of the RNA were determined using the Nanodrop (Thermo Fisher Scientific, Waltham, MA, USA) and Agilent Bioanalyzer 2100 system (Agilent Technologies, CA, USA), respectively. Then, RNA concentration was measured using $\mathrm{Qubit}^{\circledR} 2.0$ Fluorometer (Life Technologies, Carlsbad, CA, USA) and a Qubit ${ }^{\circledR}$ RNA Assay Kit. The library construction 
and sequencing were completed by Mega Genomics Ltd., Co (Beijing, China). In brief, $1 \mu \mathrm{g}$ RNA was used per sample and rRNA was removed by a NR603-VAHTS Total RNA-seq (HMR) Library Prep Kit (Vazyme Biotech Co., Ltd, Nanjing, China). The first-strand cDNA was synthesized using random hexamer primers followed by the second-strand using DNA polymerase I and RNase H. Then, the double-stranded DNA was purified by AMPure XP beads, and a poly a tail was ligated to the sequencing joint. The USER enzyme was used to degrade the cDNA strands, and the first-strand cDNA was sequenced, thereby preserving the direction of the RNA. Finally, PCR amplification was conducted and the products were purified to construct the cDNA libraries. After quantification using the Agilent BioAnalyzer 2100 system and q-PCR, the libraries were sequenced on an Illumina HiSeq $X$ Ten platform that generated 150-bp long paired-end reads.

\section{Assembling RNA Transcripts}

Reads containing the adapter sequence and low-quality reads were removed from the raw reads using Fastp software ${ }^{24}$ in the paired-end mode with default parameters, and only the high-quality clean reads were used for subsequent analyses. Clean reads were aligned to the rabbit reference genome (GCF_000003625.3_OryCun2.0_genomic.fa) along with annotated genes (GCF_000003625.3_OryCun2.0_genomic. gff) using histat2 (2.0.5) software ${ }^{25}$ with the parameters "-dta -rf-p 1-x-1-1-S File for SAM output (default:stdout)". The StringTie program ${ }^{26,27}$ was used to assembly known and novo transcripts, which guided by reference annotation genes. Cuffcompare ${ }^{28}$ was used to compare the data with known gene model to further discovered unknown genes and new exons of known genes, and to optimize the start and end positions of known genes at the same time. StringTie (1.3.3) was used to quantify transcripts and normalize the expression values (FPKM).

\section{Screening of DEGs}

Pearson's correlation coefficient $(r)^{29}$ was used to evaluate the biological-repeated correlation of samples. Differentially expressed genes (DEGs) between any two libraries were identified by DESeq (1.26.0), ${ }^{30}$ with Padjust $<0.01$ and an absolute value of the $\mid \log 2$ (fold change) $\mid \geq$ 2.0 or $\leq 1 / 2.0$ as the threshold. We performed GO (http:// www.geneontology.org/) enrichment and KEGG (https:// www.genome.jp/kegg/) pathway analysis of the DE genes using TopGO ${ }^{31}$ and KOBAS (2.0) ${ }^{32}$ software, relatively.
Significance was calculated using the Expression Analysis method and $P$ value $<0.05$ was considered significant.

\section{Validation of DEGs by q-PCR}

Primers for the coding genes and internal controls (Additional file 1) were designed using Primer-BLAST (https://www.ncbi.nlm.nih.gov/tools/primer-blast/). Total RNA was converted to cDNA by using a PrimeScript ${ }^{\mathrm{TM}}$ RT Reagent Kit containing gDNA Eraser (TAKARA, Dalian, China), and oligo (dT) and random hexamer primers. The q-PCR was performed using SYBR Premix Ex

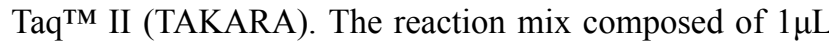
template cDNA, $0.4 \mu \mathrm{L}$ of $10 \mu \mathrm{M}$ forward and reverse primers, $5 \mu \mathrm{L}$ SYBR Premix Ex Taq ${ }^{\mathrm{TM}} \mathrm{II}$, and $3.2 \mu \mathrm{L}$ dH2O at a final volume of $10 \mu \mathrm{L}$. The reactions were performed on a Rotor gene 6000 PCR System (QIAGEN, Hiden, Germany) as follows: $95^{\circ} \mathrm{C}$ for $10 \mathrm{~s}$, followed by 40 cycles of $95^{\circ} \mathrm{C}$ for $5 \mathrm{~s}$, and $20 \mathrm{~s}$ at the $\mathrm{Tm}$ (Additional file 1). Melting curve analysis was performed from $65^{\circ} \mathrm{C}$ to $95^{\circ} \mathrm{C}$ in $1.5^{\circ} \mathrm{C}$ increments. The expression levels of genes were normalized to HPRT1 and GAPDH. Relative gene expression levels were calculated using the $2^{-\Delta \Delta \mathrm{Ct}}$ method $^{33}$ and data were expressed as mean \pm standard error of the mean (SEM).

\section{Statistical Analysis}

Statistical analysis was performed using the SPSS Statistics 20.0 (SPSS Inc., Chicago, IL, USA). The significance level was set at $P<0.05$.

\section{Results}

\section{Morphological Observation of Adipocyte Growth}

HE stained paraffin sections of the visceral adipose tissue of rabbits aged 35 (YR), 85 (MR) and 120 (TR) days were observed (Figure 1). Under the microscopic observation of 100 - and 400-fold, adipocytes were aggregated into groups based on their sizes and were either vacuolized, round, or polygonal. Due to the extrusion of lipid droplets, the flat cell nuclei could be seen at the edge of the cell. The volume of adipocyte monomers was increased with the age of the animal.

\section{Overview of RNA-Seq}

We constructed eight cDNA libraries (YR-1, YR-2, YR-3, MR-1, MR-2, TR-1, TR-2 and TR-3) from the peri-renal adipose tissues. The libraries were sequenced using the 


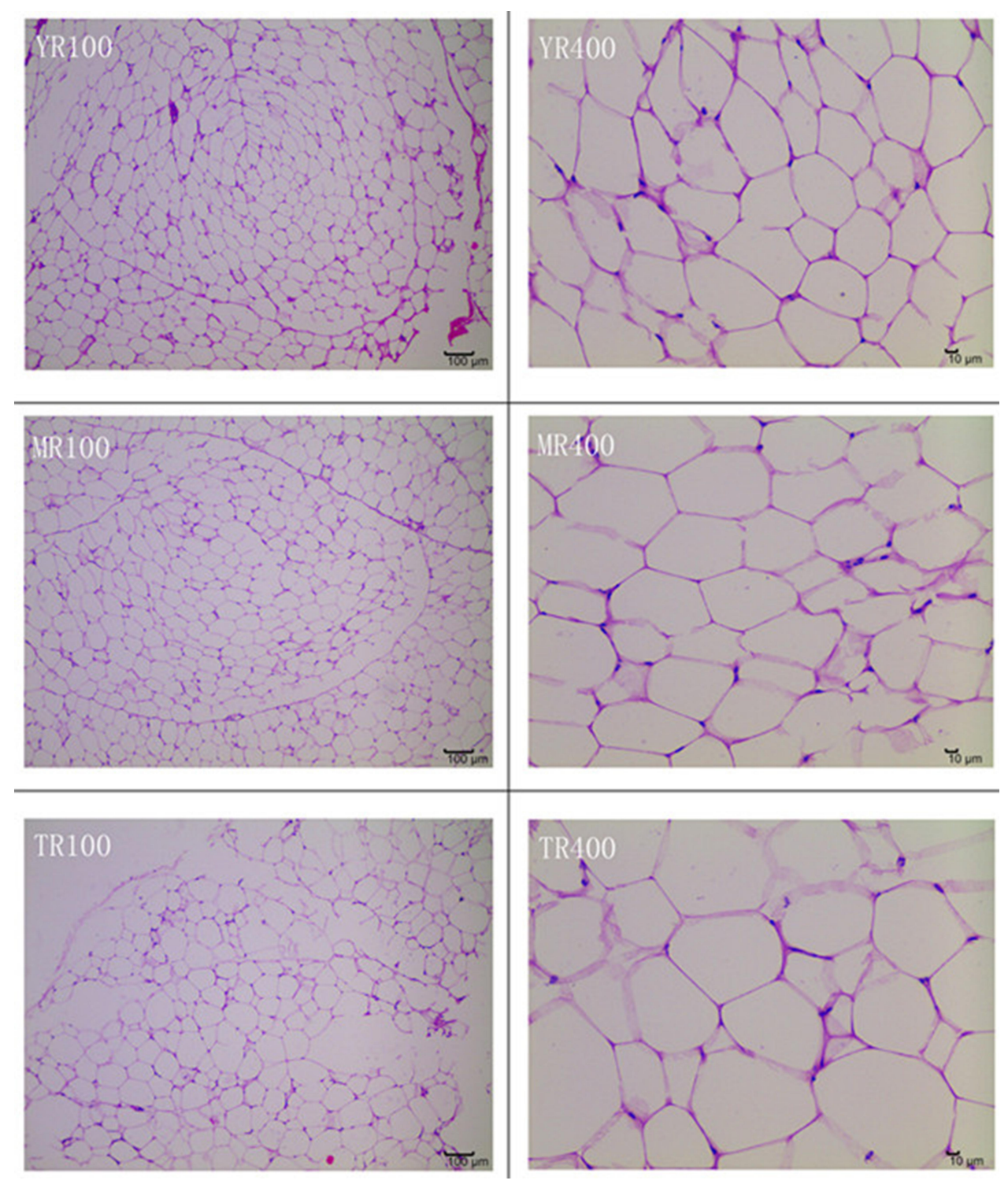

Figure I Morphological observation of visceral adipose tissue at different growth stages in rabbits.

Illumina HiSeq X Ten, and a total of $152.41 \mathrm{~Gb}$ raw reads were obtained, with an average of $19.05 \pm 0.72 \mathrm{~Gb}$ raw reads per library. After filtering adaptor sequences and low-quality reads, $120,110,494$ to $125,607,312$ clean reads were obtained in each library, with the effective ratio of each library ranging from $97.22 \%$ to $98.12 \%$ (Additional file 2). Majority (88.54-90.44\%) of clean reads mapped to the rabbit reference genome (NCBI), $85.92-87.99 \%$ of the reads had unique genomic positions (Additional file 3).

\section{Identification of DEGs}

A total of 119,502 reliable transcripts were identified from 8 libraries, including 20,303 known transcripts and 99,199 new transcripts. Among them, 98,738 transcripts were expressed in YR, 89,261 in MR and 94,863 in TR (Figure 2). The Pearson's correlation coefficient $\mathrm{R}$ of all expressed transcripts in eight samples $(n=119,502)$ results showed that the eight samples were divided into YR, MR and TR. At the same time, the biological replicates in each group were well clustered together (Figure 3). The expression levels of the coding genes were calculated by FPKM using DESeq (Additional file 4). A total of 34 DEGs were identified $(P<0.05)$ during the growth of adipose tissue (Figure 4), and 63.3\% were up-regulated and $36.7 \%$ were down-regulated. Pairwise comparison of the YR, MR and TR gene data showed that there were 25, 25 and 10 DEGs between the respective growth stages. 


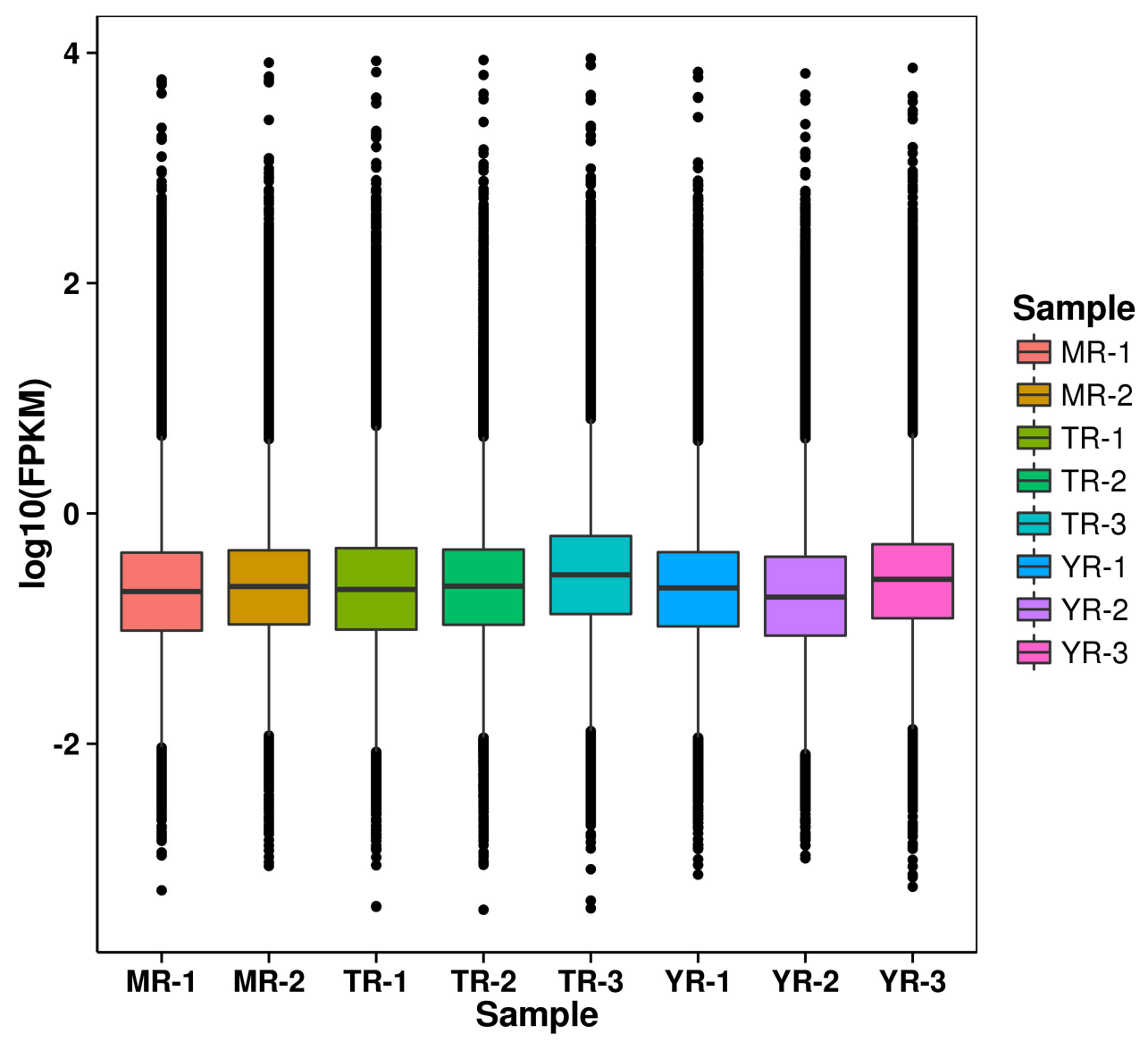

Figure 2 The analysis of FPKM Expression levels in each library.

\section{Enrichment Analysis of DEGs}

To better understand the function of 34 DEGs, we performed GO functional enrichment analysis. DEGs were enriched into $118 \mathrm{GO}$ terms, and $33 \mathrm{GO}$ terms were significant $(P<0.05)$ enrichment. Among the 33 GO terms, some terms related to lipid metabolism regulation, including acylglycerol metabolic process (GO:0006639), cellular lipid metabolic process (GO:0044255), glycerol lipid metabolic process (GO:0046486) and lipid metabolic process (GO:0006629); some DEGs such as LOC100342322 and LOC100342572's GO terms related to mobilization and decomposition of lipids to generate ATP in adipocytes, including response to reactive oxygen species (GO:0000302), monooxygenase activity (GO:0004497), response to oxidative stress (GO:0006979), and response to oxygen-containing compound (GO:1901700). In addition, ribonucleotide binding (GO:0032553), endoplasmic reticulum (GO:0005783), and nuclear outer membraneendoplasmic reticulum (GO:0042175) were significantly enriched, indicating amino acids synthesis and utilization is high during the growth of rabbit adipose tissue. It was worth noting that cell aging (GO:0007569) and aging (GO:0007568) were also significantly enriched (Figure 5).

The DEGs mapped onto 14 KEGG pathways, of which 11 KEGG pathways were significantly enriched $(P<0.05)$. These included Aminoacyl-tRNA biosynthesis (ko00970), Ribosome (ko03010), Linoleic acid metabolism (ko00591) and Biosynthesis of unsaturated fatty acids (ko01040). Furthermore, Fatty acid metabolism (ko01212), PPAR signaling pathway (ko03320) and AMPK signaling pathway (ko04152) were not significantly enriched, despite these genes being implicated in adipose growth in previous studies. This further indicates that the DEGs obtained likely play an important role during rabbit adipose growth (Figure 6).

\section{Genetic Screening of Adipose Growth-Related}

We next identified all coding genes in the transcriptome, and found 603 new genes with relevant GO terms, and 2576 new genes with KEGG pathway matches. We 


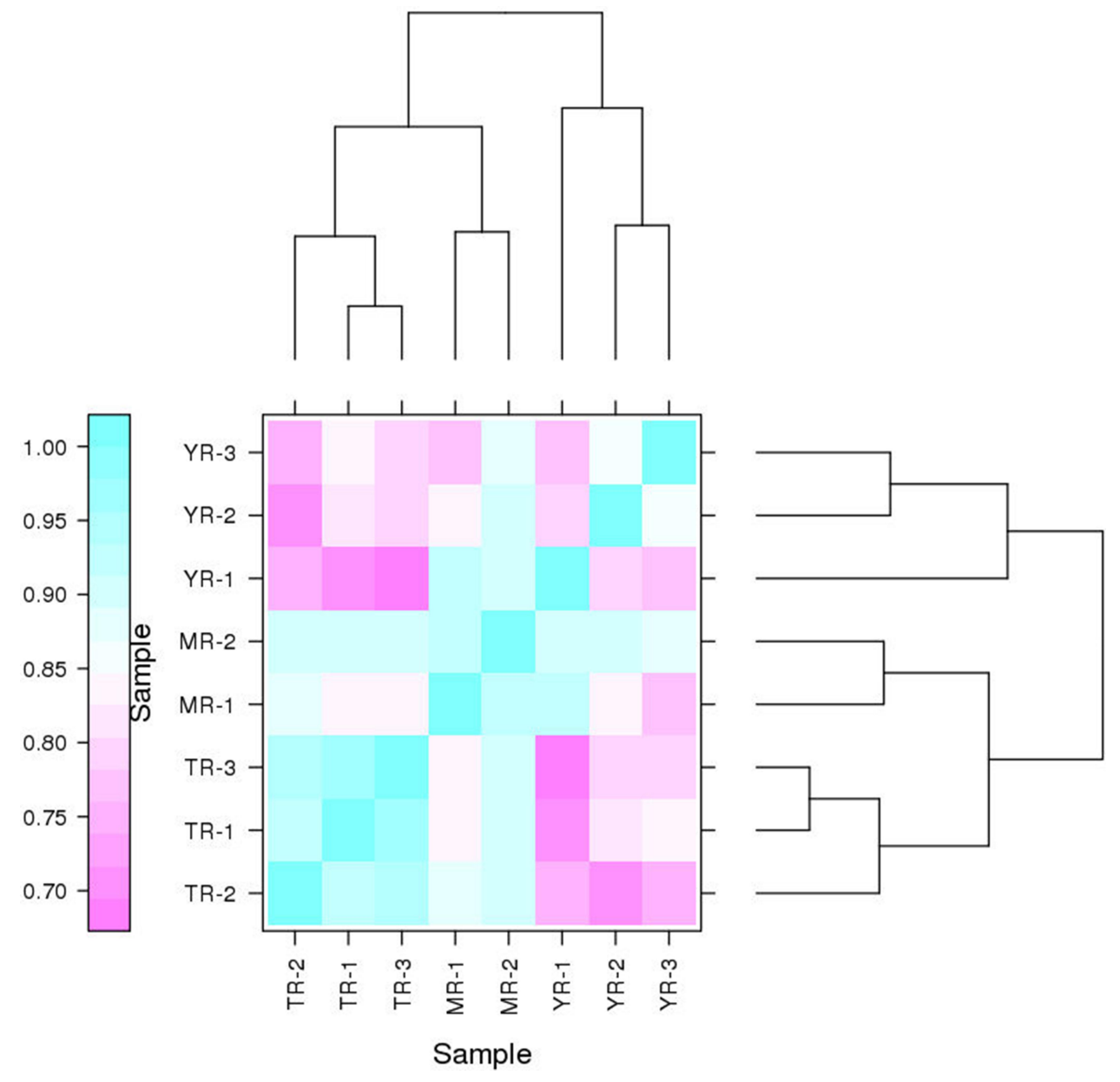

Figure 3 Hierarchical cluster analysis of all expressed transcripts $(n=1 \mid 9,502)$ in 8 libraries.

selected the functional pathway information related to growth and development of adipocytes as the screening criteria, including GO terms such as fatty acid metabolism, reactive oxygen metabolism and cAMP-dependent protein kinase inhibitor activity and KEGG pathways such as PPAR signaling, MAPK signaling, fat digestion and absorption. In total, 133 protein-coding genes which play a role in adipose growth and development were identified, including fatty acid binding protein $2(F A B P 2)$, acyl-CoA synthetase long chain family member 5 (ACSL5) and apolipoprotein B $(A P O B)$ (Additional File 5).

\section{Validation of DEGs}

To validate the RNA-Seq results, we randomly selected six DEGs and examined their expression patterns at the three growth stages by q-PCR. All six genes (NewGene_126260, NewGene_41572, MME, NewGene_70974, FAM43A, LOC108177184) were differentially expressed at different stages. In addition, the six genes exhibited a similar trend between the results of RNA-seq and q-PCR (Figure 7). Therefore, the FPKM obtained from RNA-seq can be reliably used to determine genes expression.

\section{Discussion}

In our study, we used RNA-seq to study changes of gene expression in the visceral adipose tissue in rabbits that were 35,85 and 120 days old. A total of $152.41 \mathrm{~Gb}$ of raw reads with read-length $150 \mathrm{bp}$ were obtained from eight libraries, which could cover the rabbit reference genome 55 times, and indicated a significant amount of reads were obtained. A total of 119,502 transcripts were obtained in the three stages, and 99,199 were unknown transcripts, included a large number of transcripts with low expression levels (FPKM 0 to 1), this showed that the library we constructed was relatively complete and that the rabbit reference genome may be incomplete. A total of 34 DEGs were identified in the three stages, and there were more up-regulated genes than downregulated. For example, these up-regulated DEGs included 


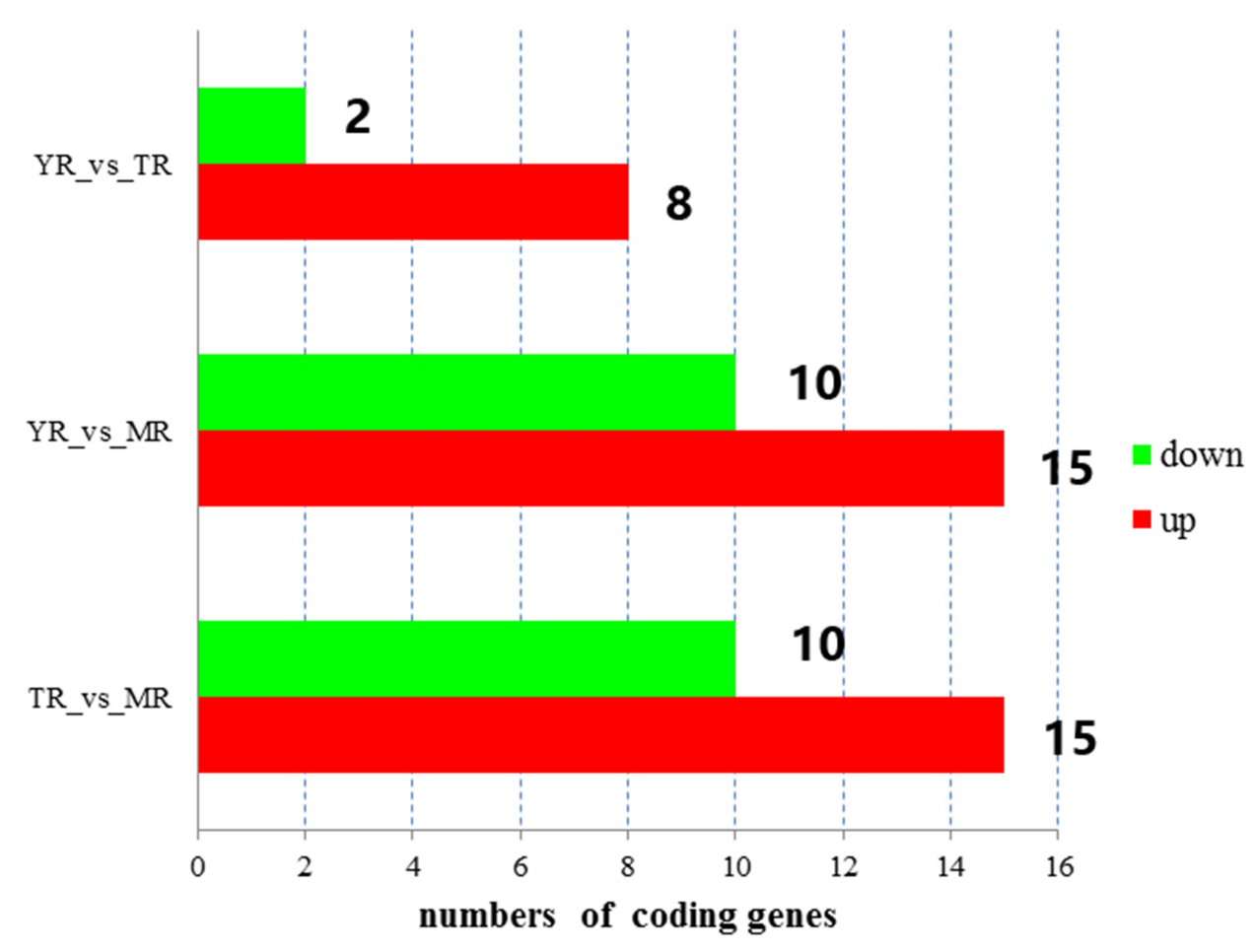

Figure 4 Numbers of up-regulated and down-regulated DEGs in the rabbit perirenal adipose at three growth stages.

FAM43A, MME and LOC108176866, while down-regulated included NewGene_70974, LOC108176300 and LOC108177184 in YR_vs_MR. This suggests that a part of the genes expression in adipocytes was gradually active during the growth of rabbits. Through functional enrichment analysis of DEGs, it was found that DEGs were significantly enriched in $33 \mathrm{GO}$ terms and 11 KEGG signaling pathways. Most of these were related to lipid metabolism, indicating that intracellular lipid droplet deposition during cell growth and maturation is regulated by a large number of genes, such as LOC100342322 and LOC100342572. Through in-depth analysis of DEGs, we found that these genes play important roles in lipid synthesis and metabolism, amino acid synthesis, endocrine and other functions during the rabbit adipose growth.

The synthesis and decomposition of triglycerides (TG) in adipocytes are constantly being carried out. When synthesis occurs more than decomposition, it promotes differentiation, adipose deposition increases, and gradually forms adipocyte with a single-chamber fat droplet. ${ }^{13}$ At this time, a large number of adipocyte marker genes are expressed, and TG synthesis, insulin sensitivity and cytokine secretion increases, thus cooperatively regulating metabolism. $^{34}$ In this study, the LOC100342322 and LOC100342572's GO terms were enriched in TG, cholesterol synthesis, lipid transport and lipid stability were up-regulated to varying degrees during the maturation of rabbit perirenal adipose tissue. Therefore, we speculated that the two genes could promote the growth of rabbit adipocytes to some extent. In addition, among 133 obesity-related genes screened, Fatty acid-binding protein $(F A B P)$, as a carrier protein of fatty acids, transports fatty acids through the cell membrane. In the fasting plasma test study of obese people with high acylation stimulating protein and high triglyceride acid, ${ }^{35}$ FABP expression increases in adipose tissue. This suggests that higher levels of $F A B P$ promote the absorption and transfer of fatty acids by adipose tissue, resulting in the accumulation of triglycerides. In addition, studies on adipocytes cultured in vitro found ${ }^{36}$ that knocking out fatty acid binding sites of $F A B P$ inhibit the formation of lipid droplets, suggesting that its function is to promote the formation of lipid droplets by binding with fatty acids. However, the high expression of $F A B P 2$ in the study suggests that $F A B P 2$ may have stabilizing effects on lipids in rabbit adipocytes.

Adipocyte hypertrophy is the main cause of visceral fat deposition, ${ }^{37}$ and adipocyte proliferation may also play a role. Peroxisome prolifrator activated receptors are important regulators of lipid metabolism and lipogenesis, ${ }^{38-40}$ 
Ontology $\square$ Biological Process $\square$ Cellular Component $\square$ Molecular Function

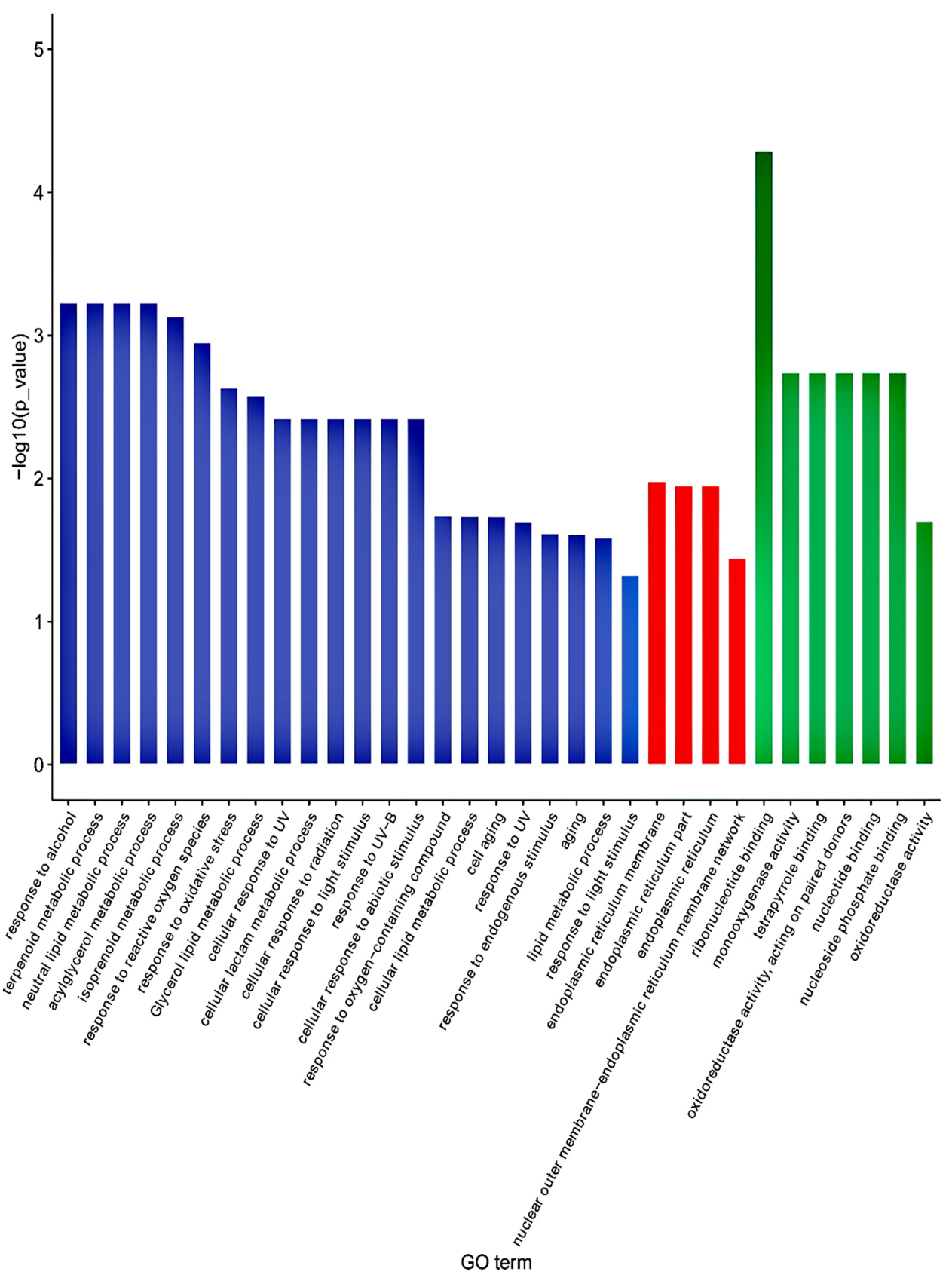

Figure 5 Gene Ontology enrichment analysis of DEGs. The significant BP, MF and CC terms in GO enrichment analysis at $p$-value $<0.05$.

which involve differentiation of pre-adipocytes and proliferation of adipocytes. ${ }^{41}$ Among 133 obesity-related genes that were screened in this study, 19 were enriched in PPARsrelated signal pathways, including $N R 1 H 3, C D 36, A C S L 5$, PRKAA2, ACSL4, ACADL, CPT2. Besides, a DE NewGene_125568 was also enriched in PPAR signal pathway. Long-chain acyl-CoA synthetase $(A C S L)$ families catalyze fatty acids, ATP and CoA to form long-chain coenzyme $\mathrm{A}$, which regulate de novo synthesis of lipids and catabolism of fatty acids. ${ }^{42,43}$ At present, five $A C S L$ subtypes can activate and guide different fatty acids to adapt to different metabolic pathways. ${ }^{44}$ ACSL4 has multiple splice subtypes with different expression patterns in mice, rats, human and pigs. ${ }^{45,46}$ Elizabeth et al ${ }^{47}$ constructed an adipocyte-specific ablation ACSL4 Ad-KO mouse using loxP Cre recombinase technology. After feeding a high-fat diet for a period of time, the lack of ACSL4 expression in adipocytes led to an increase in cell oxygen consumption and body energy consumption. 


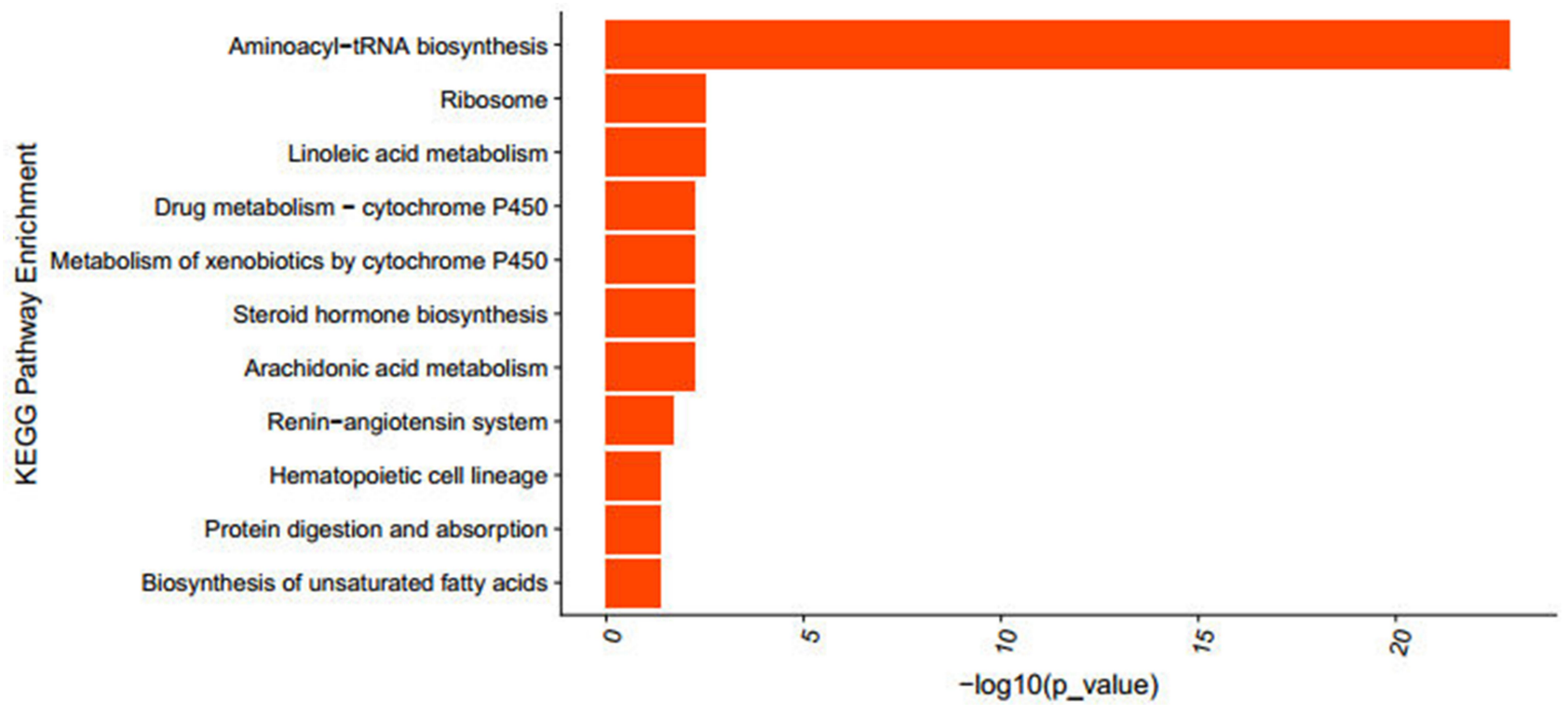

Figure 6 The KEGG pathway analysis of DEGs. The vertical axis showed the significantly enriched pathways with $p$-value $<0.05$.

ACSL5 is highly expressed in the liver, small intestine, white

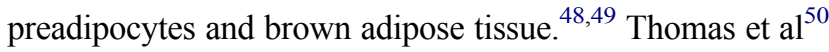
established the mouse model with impaired ACSL5 expression. Compared with normal mice, ACSL5 null mice had significantly reduced body fat content, and its fasting blood glucose and serum triglyceride were significantly reduced; therefore, impaired ACSL5 expression reduces fat and triglyceride absorption rate, and increases energy consumption and number of beige adipocytes. Based on our transcriptomic analysis, genes (ACSL4, ACSL5, NewGene_125568, LOC100342322, LOC100342572, FABP2) involved in lipid synthesis and metabolism are likely involved in rabbit adipose growth.

Adipose tissue is a frequently underestimated endocrine organ and energy homeostasis regulator, ${ }^{51,52}$ which plays a key role in amino acid regulation through circulating regulation of branched chain amino acids (BCAAs) levels. ${ }^{53}$ BCAAs include leucine, isoleucine and valine, which are metabolic signals of obesity and diabetes that can directly act on adipocytes, affect fat metabolism and reduce fat. ${ }^{54,55}$ The secretion of alanine and glycine can be detected in mammalian cell cultures, and thus such amino acids can serve as a cell nitrogen pool. ${ }^{56}$ Scott et $\mathrm{al}^{57}$ found that propionyl coenzyme A was a primer for fatty acid synthetase to produce odd chain fatty acids, and BCAA was involved in the production of all fatty acids. Duan et $\mathrm{al}^{58}$ took pigs as the research object and found that adding dietary BCAAs in different ratios to the diet reduced the total weight of pig fat and increased the concentration of adiponectin, which could regulate the function of adipose tissue, including fatty acid synthesis, transportation, oxidation, lipolysis and secretion of adipokines. In GO terms, DEGs were not only significantly enriched in nucleoside phosphate binding, the endoplasmic reticulum, and the nuclear outer membrane-endoplasmic reticulum membrane network but also enriched related to cellular nitrogen compound metabolism processes, protein metabolism processes, peptidase activity, hydrolase activity. KEGG pathway analysis showed that aminoacyl-tRNA biosynthesis, ribosome, reninangiotensin system, protein digestion and absorption were closely related to amino acid synthesis and metabolism. The above results speculated that the anabolism of amino acids in adipocytes by these DEGs such as LOC108177184 and LOC100353987 could be responsible for the less fat deposition during the rabbit growth. In addition, the GO terms of MME were significantly enriched in cell aging and aging, which indicated that DEGs related to cell aging and apoptosis might play a role in regulating adipose deposition in rabbits.

\section{Conclusions}

In the present study, we provide a global view of the adipose tissue transcriptome of three growth stages of rabbits, where we identified new putative protein-coding genes and the expression levels of known genes in adipose tissue. A total of 34 genes were found to be differentially expressed between groups and 133 obesity-related genes were screened. These genes belonged to molecular functions related to lipid, fatty acid metabolism, and PPAR signaling. Hence, this study will allow for the 

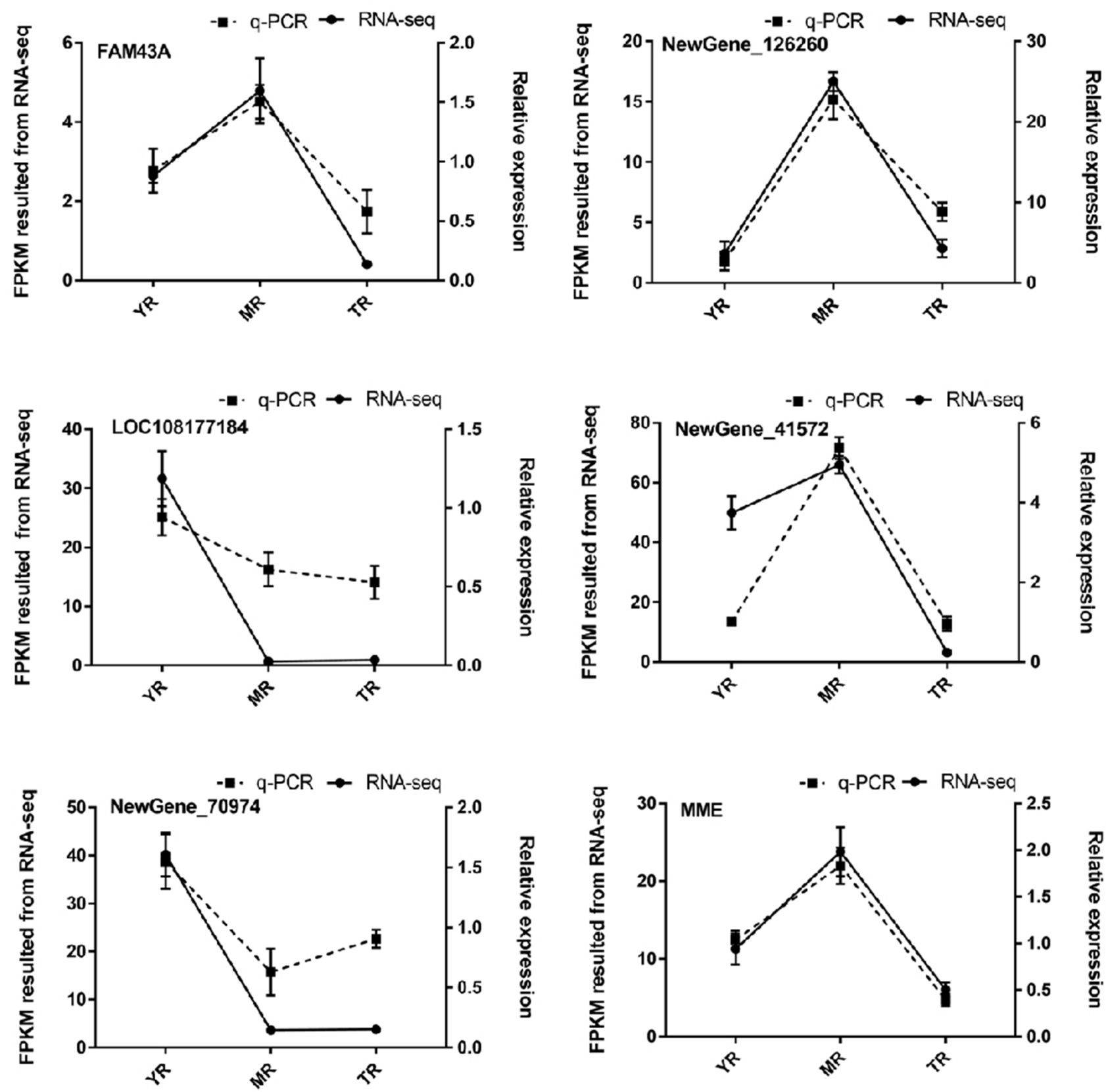

Figure 7 Validation of six DEGs by q-PCR.

identification of candidate genes for subsequent studies on rabbit genetics and adipose regulation, and provide an animal model to obesity-related diseases in humans.

\section{Abbreviations}

DEGs, differentially expressed genes; ADSCs, human adipose-derived stem cells; FPKM, fragments per kilobase of transcript per million fragments mapped; GO, Gene Ontology; KEGG, Kyoto Encyclopedia of Genes and Genomes; BCAAs, branched chain amino acids; ACSL,
Long-chain acyl-CoA synthetase; TG, triglycerides; PPARs, peroxisome proliferator activated receptors.

\section{Data Sharing Statement}

All data generated or analyzed during this study are included in this published article.

\section{Ethics}

The protocol of this study was approved by the Sichuan Agricultural University experimental animal operation 
regulations and welfare management committee and the Biological Studies Animal Care and Use Committee, Sichuan Province, China. Animal handling and care were carried out throughout the experiment according to Chinese National Guidelines for Experimental Animal Welfare. Rabbits had free access to food and water under normal conditions and were humanely sacrificed as necessary to ameliorate suffering.

\section{Consent for Publication}

Not applicable.

\section{Acknowledgments}

We thank the staff at our laboratory for their ongoing assistance. We also thank Zhou-lin Wu for insightful feedback on the study.

\section{Funding}

This work was supported by National rabbit industry technology system (CARS-43-A-2), Academic New Seedling Project of Guizhou Medical University (19NSP070) and Doctor Start-up Project of Guizhou Medical University (YJ2019005).

\section{Disclosure}

The authors report no conflicts of interest for this work.

\section{References}

1. Cullere M, Zotte AD. Rabbit meat production and consumption: state of knowledge and future perspectives. Meat Sci. 2018;143:137-146. doi:10.1016/j.meatsci.2018.04.029

2. Martínez-Álvaro M, Hernández P, Agha S, Blasco A. Correlated responses to selection for intramuscular fat in several muscles in rabbits. Meat Sci. 2018;139:187-191. doi:10.1016/j.meatsci. 2018.01.026

3. Seok Jin H, Hyuk LS, Sung Min J, et al. Vocal fold wound healing after injection of human adipose-derived stem cells in a rabbit model. Acta Otolaryngol. 2011;131(11):1198-1204. doi:10.3109/ 00016489.2011.599816

4. Chun HJ, Kim YS, Kim BK, et al. Transplantation of human adipose-derived stem cells in a rabbit model of traumatic degeneration of lumbar discs. World Neurosurg. 2012;78(3-4):364-371. doi:10.1016/j.wneu.2011.12.084

5. Xinhai Y, Peng Z, Shaobo X, Yipin X, Jian T, Guangpeng L. Adiposederived stem cells alleviate osteoporosis by enhancing osteogenesis and inhibiting adipogenesis in a rabbit model. Cytotherapy. 2014;16 (12):1643-1655. doi:10.1016/j.jcyt.2014.07.009

6. Numata H, Nakase J, Oshima T, Tsuchiya H. Effectiveness of adhering adipose-derived stem cells to defective cartilage in promoting cartilage regeneration in a rabbit model. Arthroscopy. 2019;35(9):2619-2626. doi:10.1016/j.arthro.2019.03.018

7. Ryan DH. The relationship of waist circumference and BMI to visceral, subcutaneous, and total body fat: sex and race differences. Obesity. 2012;19(2):402-408.
8. Bauer S, Weigert J, Neumeier M. Low-abundant adiponectin receptors in visceral adipose tissue of humans and rats are further reduced in diabetic animals. Arch Med Res. 2010;41(2):75-82. doi:10.1016/j. arcmed.2010.02.010

9. Wajchenberg BLO. Subcutaneous and visceral adipose tissue: their relation to the metabolic syndrome. Endocr Rev. 2000;21(6):697.

10. Luong Q, Huang J, Lee KY. Deciphering white adipose tissue heterogeneity. Biology. 2019;8(2):23. doi:10.3390/biology8020023

11. Rutter H, Bes-Rastrollo M, Henauw SD, et al. Balancing upstream and downstream measures to tackle the obesity epidemic: a position statement from the european association for the study of obesity. Obes Facts. 2017;10(1):61. doi:10.1159/000455960

12. Gregoire FM. Adipocyte differentiation: from fibroblast to endocrine cell. Expbiolmed. 2001;226(11):997.

13. Fève B. Adipogenesis: cellular and molecular aspects. Best Pract Res Clin Endocrinol Metab. 2005;19(4):483-499. doi:10.1016/j.beem.2005. 07.007

14. Desando G, Cavallo C, Sartoni F, et al. Intra-articular delivery of adipose derived stromal cells attenuates osteoarthritis progression in an experimental rabbit model. Arthritis Res Ther. 2013;15(1):R22. doi: $10.1186 / a r 4156$

15. Yu L, Zhang R, Li P, et al. Erratum to: traditional Chinese medicine: salvia miltiorrhiza enhances survival rate of autologous adipose tissue transplantation in rabbit model. Aesthetic Plast Surg. 2015;39(6):992. doi:10.1007/s00266-015-0585-6

16. Wang Z, Gerstein M, Snyder M. RNA-Seq: a revolutionary tool for transcriptomics. Nat Rev Genet. 2009;10(1):57-63. doi:10.1038/ $\operatorname{nrg} 2484$

17. Wang Z, Li Q, Chamba Y, et al. Correction: identification of genes related to growth and lipid deposition from transcriptome profiles of pig muscle tissue. PLoS One. 2017;12(2):e0172930. doi:10.1371/ journal.pone. 0172930

18. Martínez DPL, Arana A, Alfonso L, Mendizábal JA, Soret B, Te Pas MFW. Adiposity and adipogenic gene expression in four different muscles in beef cattle. PLoS One. 2017;12(6):e0179604. doi:10.1371/journal.pone. 0179604

19. Zhang YY, Wang HB, Wang YN, et al. Transcriptome analysis of mRNA and microRNAs in intramuscular fat tissues of castrated and intact male Chinese Qinchuan cattle. PLoS One. 2017;12(10):e185961.

20. Lin PP, Gao ZY, Yuan YN, et al. Developmental expression of PPAR $\alpha$ and PPAR $\gamma$ mRNA in adipose tissues of different fat-tailed sheep. Chin J Anim Vet Sci. 2012;43(9):1369-1376.

21. Li B, Qiao L, An L, et al. Transcriptome analysis of adipose tissues from two fat-tailed sheep breeds reveals key genes involved in fat deposition. BMC Genom. 2018;19(1):338. doi:10.1186/s12864-018-4747-1

22. Zhang T, Zhang X, Han K, et al. Genome-wide analysis of lncRNA and mRNA expression during differentiation of abdominal preadipocytes in the chicken. Genesgenetics. 2017;7(3):953-966. doi:10.1534/ g3.116.037069

23. Milisits G, Lévai A, Andrássybaka G, Romvári R. In vivo examination of fat deposition in growing rabbits selected for high and low body fat content. Agric Conspec Sci. 2003;68(3).

24. Shifu C, Yanqing Z, Yaru C, Jia G. fastp: an ultra-fast all-in-one FASTQ preprocessor. Bioinformatics. 2018;34(17):i884-i890. doi:10.1093/bioinformatics/bty560

25. Kim D, Langmead B, Salzberg SL. HISAT: a fast spliced aligner with low memory requirements. Nat Methods. 2015;12(4):357-360. doi:10.1038/nmeth.3317

26. Franceschini A, Szklarczyk D, Frankild S, et al. STRING v9.1: protein-protein interaction networks, with increased coverage and integration. Nucleic Acids Res. 2013;41(D1):D808-D815. doi:10.1093/nar/gks1094

27. Pertea M, Pertea GM, Antonescu CM, Chang TC, Mendell JT, Salzberg SL. StringTie enables improved reconstruction of a transcriptome from RNA-seq reads. Nat Biotechnol. 2015;33 (3):290-295. doi:10.1038/nbt.3122 
28. Ghosh S, Chan CKK. Analysis of RNA-Seq data using TopHat and Cufflinks. Plant Bioinform. 2016.

29. Schulze SK, Kanwar R, Gölzenleuchter M, Therneau TM, Beutler AS. SERE: single-parameter quality control and sample comparison for RNA-Seq. BMC Genom. 2012;13(1):524. doi:10.1186/1471-2164-13-524

30. Leng N, Dawson JA, Thomson JA, et al. EBSeq: an empirical Bayes hierarchical model for inference in RNA-seq experiments. Bioinformatics. 2013;29(8):1035-1043. doi:10.1093/bioinformatics/ btt087

31. Aibar S, Fontanillo C, Droste C, Rivas JDL. Functional Gene Networks: R/Bioc package to generate and analyse gene networks derived from functional enrichment and clustering. Bioinformatics. 2015;31(10):1686-1688. doi:10.1093/bioinformatics/btu864

32. Xie C, Mao X, Huang J, et al. KOBAS 2.0: a web server for annotation and identification of enriched pathways and diseases. Nucleic Acids Res. 2011;39(suppl_2):W316-W322.

33. Livak KJ, Schmittgen TD. Analysis of relative gene expression data using real-time quantitative PCR and the 2(-Delta Delta $\mathrm{C}(\mathrm{T})$ ) method. Methods. 2001;25(4):402-408. doi:10.1006/meth.2001.1262

34. Mikkelsen TS, Xu Z, Zhang X, et al. Comparative epigenomic analysis of murine and human adipogenesis. Cell. 2010;143 (1):156-169. doi:10.1016/j.cell.2010.09.006

35. Maclaren RE, Cui W, Lu HL, Simard S, Cianflone K. Association of adipocyte genes with ASP expression: a microarray analysis of subcutaneous and omental adipose tissue in morbidly obese subjects. BMC Med Genomics. 2010;3(1):3. doi:10.1186/1755-8794-3-3

36. Wonbaek Y, Jaeseob L, Sangeun P, et al. Albumin expression is required for adipocyte differentiation of 3T3-L1 cells. Biochem Biophys Res Commun. 2010;397(2):170-175. doi:10.1016/j. bbrc.2010.05.067

37. Cartwright AL. Adipose cellularity in Gallus domesticus: investigations to control body composition in growing chickens. J Nutr. 1991;121(9):1486. doi:10.1093/jn/121.9.1486

38. Fajas L, Fruchart JC, Auwerx J. Transcriptional control of adipogenesis. Curr Opin Cell Biol. 1998;10(2):165. doi:10.1016/ S0955-0674(98)80138-5

39. Guan YF, Breyer MD. Peroxisome proliferator-activated receptors (PPARs): novel therapeutic targets in renal disease. Kidney Int. 2002;61(1):354. doi:10.1046/j.1523-1755.2002.00129.x

40. Gilde AJ, Ka VDL, Willemsen PH, et al. Peroxisome proliferator-activated receptor (PPAR) alpha and PPARbeta/delta, but not PPARgamma, modulate the expression of genes involved in cardiac lipid metabolism. Circ Res. 2003;92(5):518-524. doi:10.1161/01.RES.0000060700.55247.7C

41. Rosen E, Sarraf PA, Bradwin G, et al. PPAR gamma is required for the differentiation of adipose tissue in vivo and in vitro. Mol Cell. 1999;4(4):611-617.

42. Klett EL, Chen S, Yechoor A, Lih FB, Coleman RA. Long-chain acyl-CoA synthetase isoforms differ in preferences for eicosanoid species and long-chain fatty acids. J Lipid Res. 2017;58 (5):884-894. doi:10.1194/jlr.M072512
43. Cooper DE, Young PA, Klett EL, Coleman RA. Physiological consequences of compartmentalized Acyl-CoA metabolism. $J$ Biol Chem. 2015;290(33):20023-20031. doi:10.1074/jbc.R115.663260

44. Mashek DG, Lei OL, Coleman RA. Long-chain Acyl-Coa synthetases and fatty acid channeling. Future Lipidol. 2007;2(4):465-476. doi:10.2217/17460875.2.4.465

45. Mercade PE, Estelle J, Perez-Enciso M. Characterization of the porcine acyl-CoA synthetase long-chain 4 gene and its association with growth and meat quality traits. Anim Genet. 2010;37 (3):219-224. doi:10.1111/j.1365-2052.2006.01436.x

46. Soupene E, Kuypers FA. Mammalian long-chain Acyl-CoA synthetases. Exp Biol Med. 2008;233(5):507-521. doi:10.3181/0710MR-287

47. Killion EA, Reeves AR, Azzouny MAE, et al. A role for long-chain acyl-CoA synthetase-4 (ACSL4) in diet-induced phospholipid remodeling and obesity-associated adipocyte dysfunction. Mol Metabol. 2018;9:S2212877817309626. doi:10.1016/j.molmet.2018.01.012

48. Mashek DG, Lei OL, Coleman RA. Rat long-chain acyl-CoA synthetase mRNA, protein, and activity vary in tissue distribution and in response to diet. J Lipid Res. 2006;47(9):2004-2010. doi:10.1194/jlr. M600150-JLR200

49. Oikawa E, Iijima H, Suzuki T, et al. A novel acyl-CoA synthetase, ACS5, expressed in intestinal epithelial cells and proliferating preadipocytes. J Biochem. 1998;124(3):679-685. doi:10.1093/ oxfordjournals.jbchem.a022165

50. Bowman TA, O'Keeffe KR, D'Aquila T, et al. Acyl CoA synthetase 5 (ACSL5) ablation in mice increases energy expenditure and insulin sensitivity and delays fat absorption. Mol Metabol. 2016;5 (3):210-220. doi:10.1016/j.molmet.2016.01.001

51. Muoio DM, Newgard CB. Obesity-related derangements in metabolic regulation. Annu Rev Biochem. 2006;75(1):367. doi:10.1146/annurev. biochem.75.103004.142512

52. Noriyuki O, Parker JL, Lugus JJ, Kenneth W. Adipokines in inflammation and metabolic disease. Nat Rev Immunol. 2011;11(2):85-97.

53. Herman MA, Pengxiang S, Peroni OD, Lynch CJ, Kahn BB. Adipose tissue branched chain amino acid (BCAA) metabolism modulates circulating BCAA levels. $J$ Biol Chem. 2010;285(15):11348-11356. doi:10.1074/jbc.M109.075184

54. Liam MA, Cotter PD, Roche HM, Riitta K, Nilaweera KN. Impact of leucine on energy balance. $J$ Physiol Biochem. 2013;69(1):155-163. doi:10.1007/s13105-012-0170-2

55. Yao K, Duan Y, Li F, et al. Leucine in obesity: therapeutic prospects. Trends Pharmacol Sci. 2016;37(8):714-727. doi:10.1016/j. tips.2016.05.004

56. Woo Suk A, Antoniewicz MR. Towards dynamic metabolic flux analysis in CHO cell cultures. Biotechnol J. 2012;7(1):61-74. doi:10.1002/biot.201100052

57. Crown SB, Marze N, Antoniewicz MR, Tochtrop GP. Catabolism of branched chain amino acids contributes significantly to synthesis of odd-chain and even-chain fatty acids in 3T3-L1 adipocytes. PLoS One. 2015;10(12):e0145850. doi:10.1371/journal.pone.0145850

58. Duan Y, Li F, Guo Q, et al. Branched-chain amino acid ratios modulate lipid metabolism in adipose tissues of growing pigs. J Funct Foods. 2018;40:614-624. doi:10.1016/j.jff.2017.12.004

Diabetes, Metabolic Syndrome and Obesity: Targets and Therapy

\section{Dovepress}

\section{Publish your work in this journal}

Diabetes, Metabolic Syndrome and Obesity: Targets and Therapy is an international, peer-reviewed open-access journal committed to the rapid publication of the latest laboratory and clinical findings in the fields of diabetes, metabolic syndrome and obesity research. Original research, review, case reports, hypothesis formation, expert opinion and commentaries are all considered for publication. The manuscript management system is completely online and includes a very quick and fair peer-review system, which is all easy to use. Visit http://www.dovepress.com/testimonials.php to read real quotes from published authors. 